\title{
Brain Processes and Neurofeedback for Performance Enhancement of Precision Motor Behavior
}

\author{
B. Hatfield, A. Haufler, and J. Contreras-Vidal \\ Department of Kinesiology \\ University of Maryland, College Park, MD 20742 \\ \{bhatfiel, haufler, pepeum\} @umd.edu
}

\begin{abstract}
Based on a number of empirical investigations of cerebral cortical dynamics during precision aiming tasks (i.e. marksmanship) employing electroencephalography (EEG) refinement of cortical activity and attenuation of nonessential cortico-cortical communication with the motor planning regions of the brain results in superior performance. Employment of EEG neurofeedback during the aiming period of target shooting designed to reduce cortical activation resulted in improved performance in skilled marksmen. Such an effect implies that refinement of cortical activity is causally related to performance. Recently, we examined cerebral cortical dynamics during the stress of competitive target shooting and observed increased activation and cortico-cortical communication between non-motor and motor regions relative to a practice-alone condition. As predicted, this finding was associated with degradation of shooting performance. These findings imply that neurofeedback targeted to brain regions related to emotional responding may preserve the cortical dynamics associated with superior performance resulting in improved accuracy of precision aiming performance.
\end{abstract}

Keywords: electroencephalography (EEG), psychomotor performance, cognitive neuroscience, stress, kinematics.

\section{Introduction}

\subsection{Cognitive Neuroscience of Skilled Motor Performance - Cortical Dynamics}

Based on a number of empirical investigations of cerebral cortical dynamics during precision aiming tasks (i.e. marksmanship) employing electroencephalography (EEG) we have formulated a model of psychomotor efficiency. In essence, this model posits that refinement of cortical activity and attenuation of nonessential cortico-cortical communication with the motor planning regions of the brain results in superior performance. This is likely due to "simplification" of central processes that emerges as economy and consistency of limb actions. More specifically, we have noted a remarkable relationship between left temporal and parietal activity during the aiming period such that quiescence or "relaxation" in this region is related to higher-quality performance. 


\subsection{Expert vs. Novice EEG Contrasts}

This principle was clearly supported by Haufler et al. [1]. Specifically, novice and expert marksmen were subjected to a target-shooting task as well as comparative verbal (i.e., word recognition) and spatial tasks (i.e., dot localization), with which the groups were similar in terms of experience, while recording EEG. The verbal and spatial tasks were also performed in the shooting stance posture. As shown in the three panels below, lower cortical activation levels in the cerebral cortex were observed in the experts during the aiming period of shooting, as measured by gamma $40-\mathrm{Hz}$ power, while no differences were revealed during the comparative tasks. The left-sided and middle panels of Figure 1 represent comparative log-transformed gamma power (36-44 Hz), which is also positively related to cortical activation, from the averaged homologous frontal (Panel $A$ ) and the averaged homologous temporal regions $($ Panel $B)$. The group differences in activation associated with the frontal region also suggest that the experts were less reliant on effortful executive processing (i.e., planning and coordinating processes) as compared to their novice counterparts. Experts also revealed significantly lower beta power, a spectral band that is positively related to cortical activation, during shooting while, again, no differences were noted during the verbal and spatial tasks. Panel $C$ shows that the experts exhibited higher levels of alpha power $(8-12 \mathrm{~Hz})$ at site $\mathrm{T} 3$ as well as lower levels of beta and gamma power. Collectively, the results clearly show task-specific relaxation in the cortex of the expert marksman.
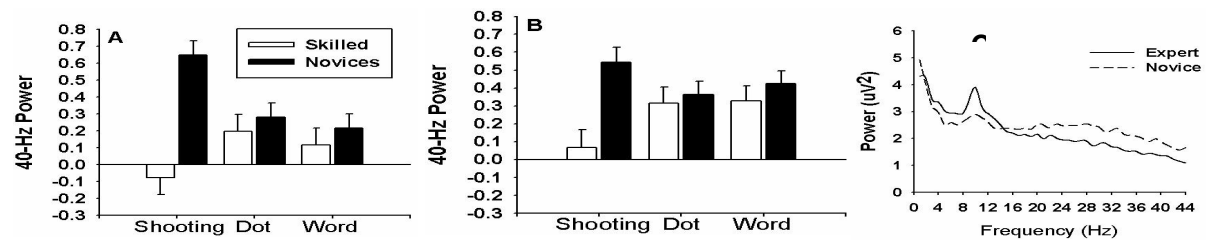

Fig. 1. Expert novice contrasts of EEG spectral power: (A) bilateral frontal gamma power (F3, F4), (B) bilateral temporal gamma power (T3, T4), (C) spectral power at T3 during shooting

\subsection{Stress and Cortical Dynamics}

Although these expert-novice contrasts and training studies did not involve direct manipulations of psychological stress, they do provide support for the notion that the individual who is highly skilled and focused shows suppression of task-irrelevant associative activity and concomitant activation of task-relevant processes [2]. The notion of mental economy is a fundamental building block of the neurobiological model of superior performance and the antithesis to the state observed during anxiety. The novice or one who is marked by a lack of confidence and much uncertainty of their performance would theoretically exhibit hyperactivity of numerous cortical processes. In this regard Kerick, Hatfield and Allender [3] recently observed a positive relationship between cortical activity (i.e., alpha suppression) and cognitive load in a study of U. S. Marines executing a target-shooting task. 
Cortical coherence and shooting performance. Additional insight can be attained into the neurobiology of the skilled performance state by examination of functional interconnectivity or cortico-cortical communication between specified topographical regions of the brain. Such "networking" activity can be quantified by deriving coherence estimates between selected pairs of electrodes or recording sites [4]. In a recent study Deeny et al. [5] assessed inter-electrode coherence between motor planning $(\mathrm{Fz})$ and association areas regions of the brain during skilled marksmanship by monitoring EEG at sites F3, F4, T3, T4, P3, Pz, P4 as well as the motor cortex (C3, Cz, C4) and visual areas (O1 and $\mathrm{O} 2)$. Coherence was assessed during a 4-second aiming period just prior to trigger pull in two groups of participants who differed in competitive performance history (experts and non-experts). The two groups were equally experienced (approximately 18 years of practice), but the "experts' consistently scored higher under the stress of competition. Figure 2 illustrates the left hemisphere Fz-F3, Fz-C3, Fz-P3, Fz-T3, and Fz-O1 coherence estimates contrasted between the two groups. A significant difference between the groups was detected for the Fz-T3 alpha band coherence, as experts revealed significantly lower values, although no other differences were observed for either the left or right hemisphere. The general lack of group differences in cortical networking seems reasonable as both were similarly experienced with the task and challenged in a similar manner. The Fz-T3 results suggest that experts limited communication between verbal-analytic and motor control processing, thereby simplifying motor planning and performing in a more accurate and consistent manner.

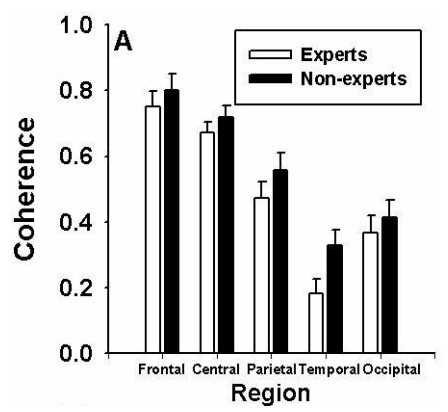

Fig. 2. The left hemisphere Fz-F3, Fz-C3, Fz-P3, Fz-T3, and Fz-O1 coherence estimates contrasted between the two groups is illustrated

Collectively, the results of these studies suggest that superior performance is marked by mental economy, particularly of analytical associative processes, and that pruning of excessive cortico-cortical communication between such processes and motor regions underlies enhancement and consistency of psychomotor (shooting) performance and raise the prediction that a reversal of these patterns would be observed with the imposition of stress.

\subsection{Affective Neuroscience - Brain Processes during Emotion}

Bear, Conners, and Paradiso [6] recently summarized the neural structures involved in a system or circuit, which mediates the psychological and physiological response to 
stress. Generally, the stress response is orchestrated by the limbic system but the central components of this functional circuit are the amygdalae, small almond-shaped structures located bilaterally and anterior to the hippocampi on the inferior and medial aspect of the temporal lobes [6]. Multiple sensory pathways converge in the basal lateral nuclei of the amygdalae so that environmental events are immediately processed. Depending on the valence of the stimuli, the lateral nuclei then communicate with the central nucleus in each amygdala and subsequent connections travel to critical forebrain, brainstem, autonomic, and endocrine structures that mediate the expression of emotion. Specifically, there are interconnections from the central nuclei to the (1) hypothalamus, which results in sympathetic arousal and stimulation of stress hormones via the hypothalamic-pituitary-adrenocortical (HPA) axis, (2) the periaqueductal grey, which results in motor responses, and (3) the cingulate cortex, which results in additional cortico-cortical communication with neocortical association regions such as the temporo-parietal regions.

In this manner orchestrated sequelae occur in response to a stressful environment, which, collectively, can change the performer's mental and physical state in a profound manner. For example, heart rate and cortisol levels rise, as does muscle tension, and the soldier may concomitantly experience excessive self-talk and "too much thinking" such that their attention is compromised and the execution of normally automated psychomotor skills such as marksmanship become explicitly managed timing and coordination are then altered and likely reduced in quality while attention shrinks.

In light of the mental and physical changes that accrue, the activation of the amygdalae serves as a pivotal event in the manifestation of stress and the control of activity in the amygdalae would exact a powerful influence on the performer's mental and physical state. Beyond the structures and processes outlined by Bear et al. [6] a critical component of the neurobiology of fear Importantly, the anterior cortical regions have extensive anatomical connections with several subcortical limbic structures implicated in emotional behavior, particularly the amygdala. Davidson and colleagues $[7,8]$ have generated a significant body of literature that clearly shows a positive association between left frontal activation and positive affect while relative right activation is associated with negative affect [7]. Although the lateralization of frontal activation is robustly related to the valence of emotion as described above, recent evidence points to a more fundamental association such that left frontal activation mediates approach-oriented behavior while right frontal activation is associated with avoidance or withdrawal-oriented behavior [8,9]. For example, left frontal activation is manifest during hostile behavior, which is certainly not a positive affective state, but most definitely involves approach toward an intended target. Whether positive in nature, approach-oriented, or a combination of the two dimensions, it would appear that such a neurobiological state would be highly adaptive for the individual who must control his/her arousal level while actively engaged with challenging tasks while under great mental stress. Therefore, cortical activation in the frontal region provides an opportune target for neurofeedback training to enable a heightened level of executive control over emotional response and task engagement during challenge. 


\subsection{Model of Stress-Induced Cortical Dynamics}

Figure 3 below provides a model of the processes and outcomes underlying stress reactivity and integrates affective and cognitive activity with psychomotor performance. A central tenet is that lack of executive control over subcortical processes would result in heightened emotional influence (limbic structures) that, in turn, disrupt higher cortical association processes that resulting in alterations in the activation of the motor loop - the fronto-basal ganglia structures that initiate and execute movement. Such disregulation interferes with attention and the motor loop connections (i.e., basal ganglia) to the motor cortex that largely control corticospinal outflow and the resultant quality of motor unit activation [10]. Excessive networking in the cortex may result in undesirable alterations in information processing as well as inconsistency of motor performance. In this manner the motor cortex becomes "busy" with excessive input from limbic processes via increased neocortical activity in the left hemisphere then inconsistent motor behavior would likely result [5].

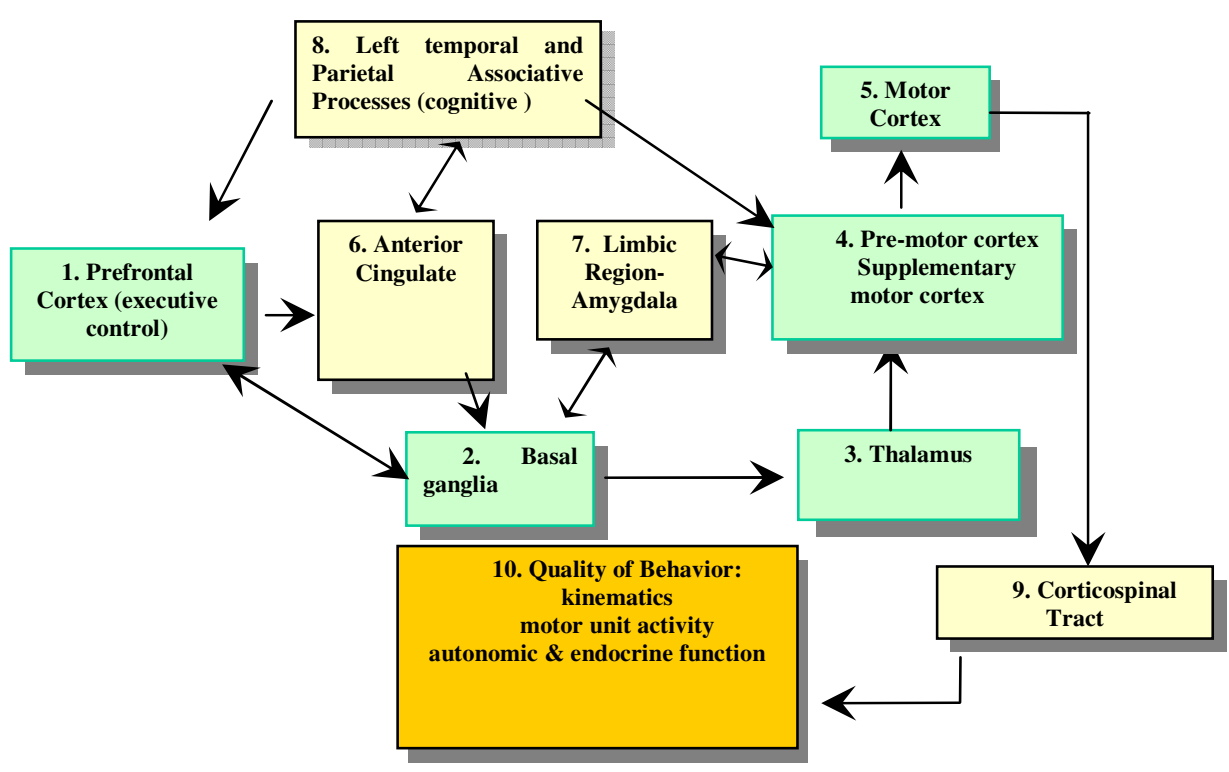

Fig. 3. Model of stress-induced cortical dynamics

Refinement or economy of cortical activation would more likely result in enhanced attention and smooth, fluid, graceful, and efficient movement. Any reduction of associative networking with motor control processes would also help to reduce the complexity of motor planning and should result in greater consistency of performance.

According to this model individuals under high stress will exhibit reductions in prefrontal asymmetry (box 1) compared to a low-stress condition implying a lack of executive control over the fronto-meso-limbic circuit. Consequently, participants will experience heightened activation of the limbic region (amygdala) (box 7). The resultant emotional reactivity, in turn, will result in EEG alpha desynchrony particularly in the left temporal (T3) and parietal (P3) regions (box 8) along with increased 
cortico-cortical communication between these regions and the motor planning centers (box 4). Such disregulation of the cerebral cortex will be expressed as inconsistent input to the motor loop (boxes 2 - 5) resulting in inconsistent corticospinal output and shooting performance (motor unit activity - trigger pull - boxes 9 and 10). It is well established that attention capacity shrinks with arousal and, consistent with this notion, the excessive cortico-cortical networking during heightened stress, as proposed here, would compromise information processing. In addition, cardiovascular activity (vagal tone) will be inversely related to the activity in the CNS such that vagal tone will be reduced in the high-stress condition. Cortisol levels will rise. The magnitude of change specified in the model will be related to degradation in shooting performance (i.e., slower and inaccurate).

\section{Methods}

\subsection{Participants}

Members of the Reserve Officers Training Corps (ROTC) participated in the study. Subjects were healthy non-smokers, between the ages of 18 and 22 years, who were right-handed and exhibited ipsilateral eye-dominance. All subjects provided informed consent, health history, and demographic information.

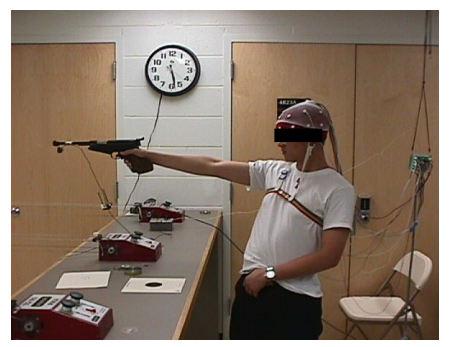

Fig. 4. Illustration of pistol shooting task

\subsection{Procedures}

Subjects completed two test sessions varying in stress and mental challenge. Figure 4 above illustrates the basic recording strategy of EEG monitoring during target shooting. Shooting scores were calculated as percentage accuracy by summing the point value of each target hit, dividing by the total points possible, and multiplying by 100 . Concentric circles on the target range from 1 to 10 , with 10 being the highest possible point total on any given shot (i.e., the bullseye).

During the low-stress condition subjects performed alone in a non-competitive state and in the absence of any time constraints to execute 40 shots. During highstress the participants competed within a sanctioned in-house match against an opponent in an adjacent lane and under the constraint of time pressure. Specifically, the 16 participants were evenly split into two teams and the scores posted during competition were publicly displayed and entered into a composite score to determine the winning team. The order of conditions was counterbalanced. 


\subsection{Psychophysiological Monitoring - Signal Acquisition / Processing}

High-density EEG records referenced to linked-ears were obtained via subjects wearing a 32-electrode stretch-lycra cap interfaced to high-impedance amplifiers (NeuroScan SynAmps system) supported by dedicated signal acquisition and processing software. Electrode impedance was maintained below $5 \mathrm{Kohm}$, amplification was $20,000 x$, bandpass filtering was $1-100 \mathrm{~Hz}$, and a sampling rate of $512 \mathrm{~Hz}$ was employed. EEG records contained event markers for all shots taken. Vertical eye movements were monitored and amplified 5,000x. Electrocardiographic records (ECG) were monitored continuously throughout each session to determine parasympathetic or vagal tonus. Strength of HPA axis activity was assessed by periodic sampling of salivary cortisol during each condition. Data collection was conducted in accord with procedures published by Putnam et al. [11] to reduce risk of infection.

EEG records corrected for artifact were subjected to Fast Fourier Transform to determine regional activation (power spectral composition) and frontal asymmetry of EEG alpha power. Inter-electrode coherence was assessed between association and motor planning regions. Furthermore, the EEG records were subjected to Independent Components Analysis (ICA), as described by Contreras-Vidal and Kerick [12], to: 1) conduct exploratory analyses for the reduction of movement artifact and 2) determine specified regional components (with particular emphasis on frontal and left temporal regions) and compute coherence between targeted brain regions.

\section{Findings}

Relative to the low-stress condition, the high-stress competitive challenge resulted in:

1. lower prefrontal asymmetry and heightened state anxiety

2. suppressed alpha power across the topography of the cortex (Figure 5)

3. increased number of cortical components clustered with a key component associated with poor performance during high stress located in the left temporal region indicative of greater cognitive complexity

4. increased alpha band coherence between left temporal and frontally located motor planning components

5. lower heart rate variability or withdrawal of vagal tone

6. heightened cortisol levels

7. degraded shooting performance

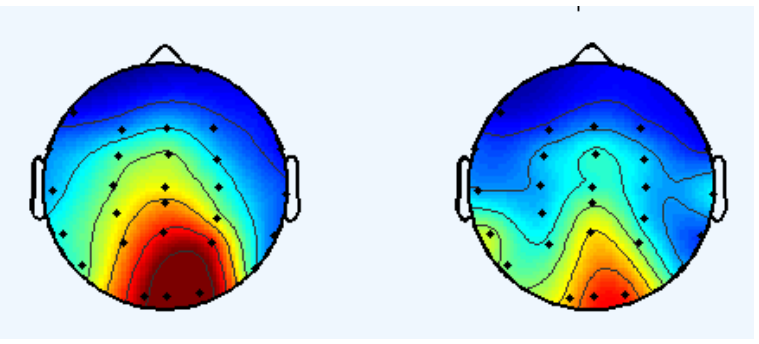

Fig. 5. Topographical EEG alpha maps during performance alone (low-stress) on left and competition (high-stress) on right at 4 seconds preceding trigger pull (Note darker shade in parietal region of low-stress condition indicating higher alpha power) 
Acknowledgements. We would like to thank Michelle Costanzo, Ron Goodman, LiChuan Lo, Jeremy Rietschel, and Mark Saffer for technical research assistance.

\section{References}

1. Haufler, A.J., Spalding, T.W., Santa Maria, D.L., Hatfield, B.D.: Neuro-Cognitive Activity During a Self-Paced Visuospatial Task: Comparative EEG Profiles in Marksmen and Novice Shooters. Biol. Psychol. 53, 131-160 (2000)

2. Hatfield, B.D., Hillman, C.H.: The Psychophysiology of Sport: A Mechanistic Understanding of the Psychology of Superior Performance. In: Singer, R.N., Hausenblas, C.H., Janelle, C.M. (eds.) Handbook of Sport Psychology, 2nd edn., pp. 362-386. John Wiley \& Sons, Inc., New York (2001)

3. Kerick, S.E., Hatfield, B.D., Allender, L.E.: Event-Related Cortical Dynamics of Soldiers During Shooting as a Function of Varied Task Demand. Aviat. Space Environ. Med. 78(5) Sect. II, B153-B164 (2007)

4. Busk, J., Galbraith, G.C.: EEG Correlates of Visual-Motor Practice in Man. Electroenceph. Clin. Neurophysiol. 38, 415-422 (1975)

5. Deeny, S., Hillman, C.H., Janelle, C.M., Hatfield, B.D.: Cortico-Cortical Communication and Superior Performance in Skilled Marksman: An EEG Coherence Analysis. J. Exerc. Sport Psychol. 25, 188-204 (2003)

6. Bear, M.F., Connors, B.W., Paradiso, M.A.: Neuroscience: Exploring the Brain. Williams \& Wilkins, Baltimore (1996)

7. Davidson, R.J.: EEG measures of cerebral asymmetry: Conceptual and methodological issues. Int. J. Neurosci. 39, 71-89 (1988)

8. Davidson, R.J.: Anxiety and Affective Style: Role of Prefrontal Cortex and Amygdala. Biol. Psychiatry 51, 68-80 (2002)

9. Jackson, D.C., Mueller, C.J., Dolski, I., Dalton, K.D., Nitschke, J.B., Urry, H.L., Rosenkranz, M.A., Ryff, C.D., Singer, B.H., Davidson, R.J.: Now You Feel it, Now You Don't: Frontal Brain Electrical Asymmetry and Individual Differences in Emotion Regulation. Psychol. Sci. 14, 612-617 (2003)

10. Grafton, S.T., Hari, R., Salenius, S.: The Human Motor System. In: Toga, A.W., Mazziotta, J.C. (eds.) Brain Mapping; The Systems, pp. 331-363. Academic Press, San Diego (2000)

11. Putnam, L.E., Johnson Jr., R., Roth, W.T.: Guidelines for Reducing the Risk of Disease Transmission in the Psychophysiology Laboratory. Psychophysiol. 29, 127-141 (1992)

12. Contreras-Vidal, J.L., Kerick, S.E.: Independent Component Analysis of Dynamic Brain Responses During Visuomotor Vdaptation. Neuroimage 21, 936-945 (2004) 